\title{
How galaxies gain and lose their angular momentum
}

\author{
Elena D'Onghia ${ }^{1} \dagger$ \\ ${ }^{1}$ Institute for Theoretical Physics, Winterthurerstrasse 190, CH-8057 Zurich, Switzerland \\ email: elena@physik.unizh.ch
}

\begin{abstract}
Spiral, fast-rotating galaxies like the Milky Way are the most common type in the Universe. One of the most pressing challenges faced by current models of galaxy formation is the origin of their angular momentum and disk. According to the standard tidal-torque theory the galactic spin is originated by tidal interactions between dark halos around galaxies and neighboring structures in the expanding Universe. We use a large cosmological N-body simulation to study the origin of possible correlations between the merging history and spin of cold dark matter halos. In particular, we examine claims that remnants of major mergers tend to have higher-than-average spins, and find that the effect is driven largely by unrelaxed systems: equilibrium dark matter halos show no significant correlation between spin and merging history. Out-of-equilibrium halos have, on average, higher spin than relaxed systems, suggesting that the virialization process leads to a net decrease in the value of the spin parameter. We present also high-resolution N-body/SPH cosmological simulations including cold gas and dark matter to investigate the processes by which gas loses its angular momentum during the protogalactic collapse phase, leading to simulated disk galaxies that are too compact with respect to the observations. We show that the gas and the dark matter have similar specific angular momenta until a merger event occurs at redshift 2 . All the gas involved in the merger loses a substantial fraction of its specific angular momentum due to tidal torques and falls quickly into the center. Dynamical friction by small infalling substructures plays a minor role, in contrast to previous claims.
\end{abstract}

Keywords. methods: N-body simulations, galaxies: formation, galaxies: haloes, cosmology: theory

\section{Introduction}

The origin of the distribution of angular momentum in disk galaxies is yet an unsolved puzzle. In the current structure formation paradigm, the net spin of a dark matter halo and of its luminous component originates in torques exerted by neighboring structures at early times. The tidal-torque theory envisions the spin of a halo as acquired during the early expansion phase, when tides are strong and when the moment of inertia that couples the material destined to collapse to external tides is large.The tidal-torque scenario also implies that the specific angular momentum acquired by the baryonic and dark matter components of a galaxy should be similar.

Recently, however, a number of authors have highlighted the possibility that mergers may play a substantial role in determining the angular momentum content of a dark matter halo. D'Onghia \& Burkert (2004), for example, argue that halos with a quiet merging history might not acquire enough angular momentum to host late-type spiral galaxies. Gardner (2001), Vitvitska et al. (2002), among others, report a significant correlation between mergers and spin and ascribe it to the large orbital angular momentum

\section{$\dagger$ Marie Curie Fellow}


associated with major mergers. Vitvitska et al. (2002), in particular, follow the evolution of the spin parameter of the most massive progenitor of several dark halos and find that the spin parameter varies with time, increasing abruptly during major mergers, and decreasing gradually during times of minor accretion. This is an intriguing result, since late major mergers are normally thought to be associated with the formation of elliptical galaxies which have long been known to be - at fixed stellar mass - deficient in angular momentum relative to spirals. It is puzzling that galaxies where rotational support is low should tend to inhabit dark halos where angular momentum is more plentiful.

On the other hand, late major-merger remnants are, by definition, halos where a significant amount of mass and angular momentum has been recently added to the halo. One might therefore expect that tides have had a chance to operate for a longer period of time on these systems, a fact that may explain their higher-than-average spins.

Is this why merger remnants are reported to have higher-than-average spins? Or do major merger remnants have instead spins comparable to those of halos that have accreted a similar amount of mass on a similar timescale through mainly smooth accretion?

\section{Spin Parameter and Halo Merging History}

The relation between mergers and spin is explored in Figure 1, where the top panel shows, for relaxed halos, the dependence of the spin parameter $\lambda$ on $f_{\text {merg }}$, the fraction of mass accreted by a halo in its single most important merger event since $z=3$. This panel shows that there is no obvious correlation between $\lambda$ and merging activity when considering systems near virial equilibrium.

Note that the relaxed halo sample includes many remnants of major merger events; $13 \%$ of them have accreted more than $25 \%$ of their mass in a single merger, and half of them have undergone at least one merger during which more than $\sim 15 \%$ of their final mass was accreted (see details in D'Onghia \& Navarro 2007). Yet, there is no obvious evidence that more massive mergers lead to higher than average spin parameters. We conclude that merging history and spin are uncorrelated in equilibrium halos. The situation is different for "unrelaxed" halos, as shown in the bottom panel of Figure 1. Here a correlation between $f_{\text {merg }}$ and $\lambda$ is clearly present. Because these systems are out of equilibrium, spin estimates are likely to fluctuate as they relax. These fluctuations must lead to a net reduction in the spin measured within the virial radius in order to explain the difference in the spin distribution of relaxed and unrelaxed halos.

\section{Angular Momentum Loss During Galactic Disk Formation}

A remarkable problem of current galaxy formation simulations in a $\Lambda$ CDM Universe is their inability to produce disk dominated galaxy. Traditionally loss of angular momentum of progenitor lumps through dynamical friction has been blamed as the cause of excessive angular momentum loss (Navarro \& Benz 1991, Navarro \& White 1994). The latest numerical simulations can form disks with realistic sizes but these disks are obtained including in the cosmological codes an arbitrary subgrid physics (Robertson et al. 2006, Governato et al. 2007). However, even in these cases no simulation has managed to form a bulgeless galaxy with a realistic, kinematically cold thin stellar disk.

We have investigated additionally the processes by which gas loses its angular momentum during the protogalactic collapse phase, leading to disk galaxies that are too compact with respect to the observations. We present a high-resolution N-body/SPH simulation of a Milky Way sized halo including cold gas and dark matter. A halo with quiet merging activity since redshift $\mathrm{z} \sim 3.8$ and with a high spin parameter is analyzed that should be 


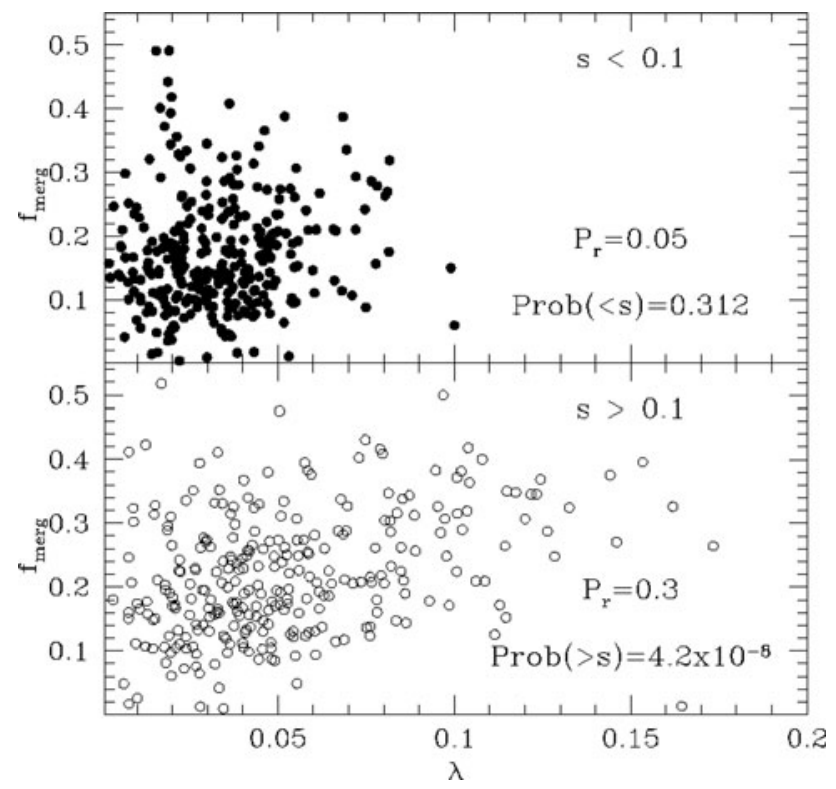

Figure 1. The fraction of mass, $f_{\mathrm{merg}}$, accreted by a halo in the single most important merger event since $z=3$, versus the spin parameter, $\lambda$, measured within the virial radius at $z=0$ for each halo in our sample. Top and bottom panels correspond to "relaxed" and "unrelaxed" halos, respectively (D'Onghia \& Navarro 2007).

an ideal candidate for the formation of an extended galactic disk. In Figure 2 we show that the gas and the dark matter have similar specific angular momenta until a merger event occurs at $\mathrm{z} \sim 2$ with a mass ratio of 5:1. All the gas involved in the merger loses a substantial fraction of its specific angular momentum due to tidal torques and dynamical friction processes falls quickly into the centre. In contrast, gas infall through small subclumps or accretion does not lead to catastrophic angular momentum loss. In fact, a new extended disk begins to form from gas that was not involved in the 5:1 merger event and that falls in subsequently. We argue that the angular momentum problem of disk galaxy formation is a merger problem: in cold dark matter cosmology substantial mergers with mass ratios of 1:1 to $6: 1$ are expected to occur in almost all galaxies. We suggest that energetic feedback processes could in principle solve this problem, however only if the heating occurs at the time or shortly before the last substantial merger event. If a large fraction of the low angular momentum gas would be ejected, late-type galaxies could form with a dominant extended disk component, resulting from late infall, a small bulge-to-disk ratio and a low baryon fraction, in agreement with observations.

\section{Final Remarks}

We examine claims that remnants of major mergers tend to have higher-than-average spins, and find that the effect is driven largely by unrelaxed systems. Equilibrium dark matter halos show no significant correlation between spin and merging history. Out-ofequilibrium halos have, on average, higher spin than relaxed systems, suggesting that the virialization process leads to a net decrease in the value of the spin parameter. We find that this decrease is due to the internal redistribution of mass and angular momentum that occurs during virialization. This process is especially efficient during major mergers, when high angular momentum material is pushed beyond the virial radius of the remnant. 


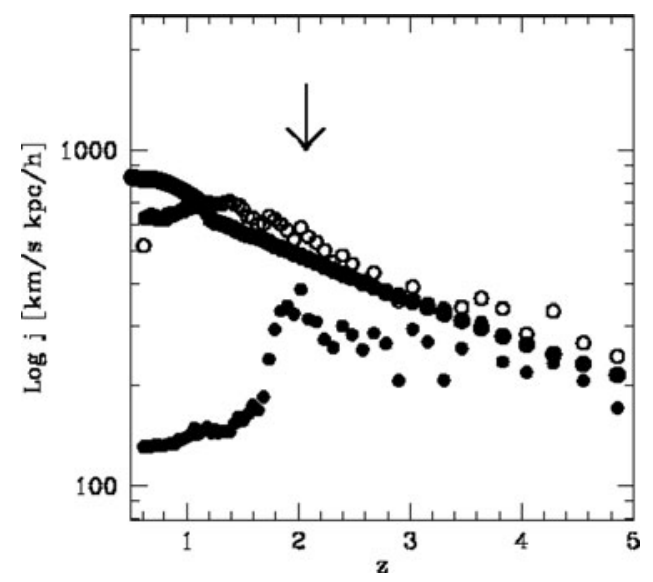

Figure 2. The angular momentum evolution of the dark matter halo (large filled circles), the gas particles placed in the inner regions (small dark circles) and the extended disk particles (open circles) of the Milky Way sized halo (D'Onghia et al. 2006)

Since such redistribution likely affects the angular momentum of baryons and dark matter unevenly, our findings question the common practice of identifying the specific angular momentum content of a halo with that of its embedded luminous component. Further work is needed to elucidate the true relation between the angular momentum content of baryons and dark matter in galaxy systems assembled hierarchically.

\section{Acknowledgements}

I wish to thank Martin Bureau and the organizers for a very fruitful Conference. I thank my collaborators: Julio Navarro, Andi Burkert, Giuseppe Murante and Sadegh Kochfar. E.D is supported by a EU Marie Curie Intra-European Fellowship under contract MEIF041569 .

\section{References}

D'Onghia, E. \& Burkert, A. 2004, ApJ, 612, L13

D'Onghia, E., Burkert, A., Murante, G., \& Khochfar, S. 2006, MNRAS, 372, 1525

D'Onghia, E. \& Navarro, J. F. 2007, MNRAS, 380, L58

Gardner, J. P. 2001, ApJ, 557, 616

Governato, F. et al. 2007, MNRAS, 374, 1479

Navarro, J. F. \& Benz, W. 1991, ApJ, 380, 320

Navarro, J. F. \& White, S. D. M 1994, MNRAS, 267, 401

Robertson, B. et al. 2006, ApJ, 645, 986

Vitvitska, M. et al. 2002, ApJ, 581, 799 